\title{
CORRIGENDUM Vol. 23
}

Caption to Fig. 1 opposite p. 150 should read:

Fig. 1. Method of counting radiation from the pods, the scaler is out of the picture. $\mathbf{P}=$ pod in counting position. $\mathbf{M}=$ metal plate enclosing the lead shield which is just visible around the pod. $\mathbf{G}-\mathbf{M}=$ the holder enclosing the Geiger-Muller tube. $\mathbf{A}=$ aluminium foil. $\mathbf{B}=$ brick screen. 